\title{
High-fat Diet Accelerates Intestinal Tumorigenesis Through Disrupting Intestinal Cell Membrane Integrity
}

\author{
Mi-Young Park ${ }^{1, *}$, Min Young Kim ${ }^{2, *}$, Young Rok Seo ${ }^{3}$, Jong-Sang Kim ${ }^{4}$, Mi-Kyung Sung ${ }^{2}$ \\ 'Department of Food and Nutrition Education, Graduate School of Education, Soonchunhyang University, Asan, ${ }^{2}$ Department of Food and Nutrition, \\ Sookmyung Women's University, ${ }^{3}$ Department of Life Science, Institute of Environmental Medicine for Green Chemistry, Dongguk University, Seoul, \\ ${ }^{4}$ School of Food Science and Biotechnology, College of Agriculture and Life Sciences, Kyungpook National University, Daegu, Korea
}

\begin{abstract}
Background: Excess energy supply induces chronic low-grade inflammation in association with oxidative stress in various tissues including intestinal epithelium. The objective of this study was to investigate the effect of high-fat diet (HFD) on intestinal cell membrane integrity and intestinal tumorigenesis in $\mathrm{ApC}^{\mathrm{Min} / \mathrm{t}}$ mice.

Methods: Mice were fed with either normal diet (ND) or HFD for 12 weeks. The number of intestinal tumors were counted and biomarkers of endotoxemia, oxidative stress, and inflammation were determined. Changes in intestinal integrity was measured by fluorescein isothiocyanate (FITC)-dextran penetration and membrane gap junction protein expression.

Results: HFD group had significantly higher number of tumors compared to ND group $(P<0.05)$. Blood total antioxidant capacity was lower in HFD group, while colonic 8-hydroxy-2'-deoxyguanosine level, a marker of oxidative damage, was higher in HFD group compared to that of ND group $(P<0.05)$. The penetration of FITC-dextran was substantially increased in HFD group $(P<0.05)$ while the expressions of membrane gap junction proteins including zonula occludens-1, claudin-1, and occludin were lower in HFD group $(P<0.05)$ compared to those in ND group. Serum concentration of lipopolysaccharide (LPS) receptor (CD14) and colonic toll-like receptor 4 (a LPS receptor) mRNA expression were significantly higher in HFD group than in ND group $(P<0.05)$, suggesting that significant endotoxemia may occur in HFD group due to the increased membrane permeability. Serum interleukin-6 concentration and myeloperoxidase activity were also higher in HFD group compared to those of ND group $(P<0.05)$.

Conclusions: HFD increases oxidative stress disrupting intestinal gap junction proteins, thereby accelerating membrane permeability endotoxemia, inflammation, and intestinal tumorigenesis.
\end{abstract}

(J Cancer Prev 2016;21:95-103)

Key Words: Apc, Mice, Colon neoplasms, High-fat diet, Permeability, Oxidative stress

\section{INTRODUCTION}

Colon cancer is the fourth leading cause of cancer deaths worldwide. ${ }^{1}$ Epidemiological studies provided evidence that environmental factors are the prime importance in the etiology of colon cancer. ${ }^{2}$ One of the most important risk factors for colon cancer is body fatness which is often resulted from imbalance between energy intake and energy expenditure. ${ }^{3.4}$ Therefore, excess dietary fat intake in association with body fat deposition has been regarded as a convincing risk factor for tumorigenesis in the colon. In fact, a chronic fat-rich diet, a typical high energy diet, could results in increased endotoxemia which is a triggering factor for inflammatory responses promoting carcinogenesis. ${ }^{5}$

Excess reactive oxygen species (ROS) generation in the obese state results in increased oxidative damage which accelerate inflammatory responses. ${ }^{6}$ Several human studies have shown that obese subjects exhibit lower systemic antioxidant defense activity than normal weight counterparts. Serum 8-hydroxy-2'-de-

Received June 6, 2016, Revised June 16, 2016, Accepted June 16, 2016

Correspondence to: Mi-Kyung Sung

Department of Food and Nutrition, Sookmyung Women's University, 100 Cheongpa-ro 47-gil, Yongsan-gu, Seoul 04310, Korea

Tel: +82-2-710-9395, Fax: +82-2-710-9453, E-mail: mksung@sm.ac.kr, ORCID: Mi-Kyung Sung, http://orcid.org/0000-0002-3575-5628

*These authors contributed equally to this work as co-first authors.

Copyright (ㄷ) 2016 Korean Society of Cancer Prevention

(c) This is an Open Access article distributed under the terms of the Creative Commons Attribution Non-Commercial License (http://creativecommons.org/licenses/by-nc/4.0) which permits unrestricted non-commercial use, distribution, and reproduction in any medium, provided the original work is properly cited. 
oxyguanosine (8-OHdG) concentration, a marker of oxidative damage, has also been found higher in pre-diabetic subjects. ${ }^{7-9}$ Increased oxidative stress is implicated in carcinogenesis through DNA, protein, and lipid oxidation, and modulation of cellular signaling. ${ }^{10-12}$ Especially, intestinal epithelial cells grow rapidly and have a high metabolic rate which makes them extremely vulnerable to oxidative DNA damage. ${ }^{13}$ Genetic alterations as well as signaling pathways to deal with the oxidative burden in epithelium are well known to be involved in the carcinogenesis.

Several lines of evidence indicate that oxidative stress also disrupts epithelial tight junctions (TJs) and increases the membrane permeability. In obese patients and animal models of obesity, intestinal permeability is shown to be increased possibly due to impairment of TJs. ${ }^{14-16}$ Although the causes and mechanisms of disruptions in TJs are not fully understood, alterations in microbiota composition and chronic low-grade inflammation may have been suggested to be linked to intestinal membrane integrity. ${ }^{17-19}$ The increase of intestinal permeability causes the development of metabolic endotoxemia with increased lipopolysaccharide (LPS) translocation into the systemic circulation. ${ }^{20}$

$A p C^{\mathrm{Min} /+}$ mice exhibit a germ-line nonsense mutation at codon 850 of adenomatous polyposis coli (APC) gene and spontaneously develop multiple polyps in the small and large intestines at the age of 10 to 12 weeks. $^{21}$ Therefore, $A p C^{\mathrm{Min} /+}$ mice are considered to be a useful animal model to study human familial adenomatous polyposis and colorectal cancers.

Although several studies have investigated possible link between systemic fat deposition and oxidative stress or inflammatory responses, few studies have been reported that the involvement of transepithelial colon permeability in high-fat diet (HFD) induced colon tumorigenesis. Therefore, in this study, we assessed the effects of HFD on oxidative damage, local inflammatory events, and intestinal integrity in association with intestinal tumorigenesis in $\mathrm{ApC}^{\mathrm{Min} /+}$ mice.

\section{MATERIALS AND METHODS}

\section{Animals and study design}

The inbred mice were originally purchased from the Jackson Laboratory (Bar Harbor, ME, USA). The $A p c^{\mathrm{Min} /+}$ pedigree was maintained by mating $A p c^{+/+}$females with $A p c^{\mathrm{Min} /+}$ males, and procedures to secure inbreeding were followed. The $A p c^{\operatorname{Min} /+}$ mice were identified by allele-specific PCR on DNA isolated from tail. All animals were housed in wire cages (4-5 mice/cage) with free access to drinking water and basal diet CE-2 (CLEA Japan Inc., Tokyo, Japan) under controlled conditions of humidity $(50 \% \pm$
$10 \%)$, lighting (12-hour light/dark cycle), and temperature $\left(23^{\circ} \mathrm{C} \pm\right.$ $2^{\circ} \mathrm{C}$ ), which were maintained until termination of the study. The composition of experimental diet was based on a modified AIN-93G diet as shown in Table 1. The fat sources of normal diet (ND, $15 \%$ of fat calories) and HFD (45\% of fat calories) were based on corn oil and lard. Mice were fed either ND or HFD for 12 weeks. Weight gain and food intake were recorded twice a week. All animals were sacrificed at the end of the study period using Zoletil and Rompun as an anesthetic. The colons were removed and rinsed in ice-cold normal saline. They were laid flat on a glass plate, and the mucosa was scraped with a slide glass. Epididymal fat pad and liver were collected immediately, rinsed in ice-cold normal saline, and weighed. Blood was collected from the inferior vena cava into EDTA tubes and centrifuged at 4,000 rpm for 20 minutes. All samples were stored at $-80^{\circ} \mathrm{C}$ until assayed. An animal protocol approved by the Institutional Animal Care and Use Committee of Sookmyung Women's University was used for all animal experiments.

\section{Tumor scoring}

At sacrifice, all organs were removed, and the small and large intestines were cut open along their longitudinal axis. The small intestine and colon were then spread flat on a microscope slide and the number of tumors was determined with an inverse light microscope with a magnification of $1 \times 10$.

Table 1. Composition of the experimental $\operatorname{diets}^{\mathrm{a}}$

\begin{tabular}{lrr}
\hline \multicolumn{1}{c}{ Variable } & ND & HFD \\
\hline Macronutrient composition & & \\
Carbohydrate (\% of energy) & 65.700 & 35.700 \\
Protein (\% of energy) & 19.300 & 19.300 \\
Fat (\% of energy) & 15.000 & 45.000 \\
Ingredient (g/kg) & & \\
Cornstarch & 404.000 & 266.500 \\
Dextrin & 134.200 & 88.500 \\
Sucrose & 101.600 & 67.100 \\
Fiber & 50.000 & 50.000 \\
Casein & 198.000 & 240.400 \\
Corn oil & 12.440 & 45.360 \\
Lard & 49.760 & 181.440 \\
Mineral mix ${ }^{\mathrm{b}}$ & 34.600 & 42.100 \\
Vitamin mix & 9.900 & 12.000 \\
L-cysteine & 3.000 & 3.700 \\
Choline bitartrate & 2.500 & 3.100 \\
Tert-butylhydroquinone & 0.014 & 0.017 \\
Total calrories/100 g & 372.800 & 452.800 \\
\hline
\end{tabular}

ND, normal diet; HFD, high-fat diet. ${ }^{a}$ Diets were prepared according to the AIN-93 G diet with slight modifications. ${ }^{b}$ Mineral mixture and vitamin mixture were prepared according to AIN-93 G diet. 


\section{Total antioxidant capacity}

Total antioxidant activity was determined by the ferric reducing antioxidant power (FRAP) assay. The FRAP reagent was prepared from $10 \mathrm{mmol} / \mathrm{L}$ 2,4,6-tri-(2-pyridyl)-1,3,5-triazine (TPTZ) solution in $\mathrm{HCl} 40 \mathrm{mmol} / \mathrm{L}$ plus $\mathrm{FeCl}_{3}(20 \mathrm{mmol} / \mathrm{L}$ ) and acetate buffer $(0.3 \mathrm{~mol} / \mathrm{L}, \mathrm{pH} 3.6)$ in a $1: 1: 10$ ratio. Freshly prepared FRAP reagent was warmed at $37^{\circ} \mathrm{C}$ for 5 minutes. Serum sample or standard $(50 \mu \mathrm{L})$ was mixed with $1.5 \mathrm{~mL}$ of FRAP reagent in a test tube and incubated at $37^{\circ} \mathrm{C}$ for 10 minutes. Then, the absorbance of the colored products (ferrous TPTZ complex) was measured at $593 \mathrm{~nm}$ and compared to the blank.

\section{Colonic 8-hydroxy-2'-deoxyguanosine concentration}

Colons were homogenized and DNA was extracted using DNA extraction kit (Nirthwest Life Science Specialities, Vancouver, BC, Canada) following the manufacture's instruction. Extracted DNA $(200 \mu \mathrm{g})$ was dissolved in $135 \mu \mathrm{L}$ of water, and $15 \mu \mathrm{L}$ of $200 \mathrm{mM}$ sodium acetate and 6 units of nuclease $P 1$ were added to the DNA solution and incubated for 30 to 60 minutes at $37^{\circ} \mathrm{C}$. Tris- $\mathrm{HCl}$ buffer ( $1 \mathrm{M}, \mathrm{pH} 7.4)$ and 2 unit of alkaline phosphatase were added and incubated for 30 to 60 minutes at $37^{\circ} \mathrm{C}$. After filtering the solution, the supernatants were used for the assay.

\section{Determination of intestinal permeability}

This measure is based on the intestinal permeability to 4,000-Da fluorescent-dextran (Sigma-Aldrich, St. Louis, MO, USA) as described previously. Briefly, 6-hour fasted mice were injected with fluorescein isothiocyanate (FITC)-dextran by gavage (600 $\mathrm{mg} / \mathrm{kg}$ body weight, $125 \mathrm{mg} / \mathrm{mL}$ ) and $120 \mu \mathrm{L}$ blood was collected from the heart 1 hour after the gavage. The blood was centrifuged at $4^{\circ} \mathrm{C}, 4,000 \mathrm{rpm}$, for 3 minutes. Plasma was diluted in an equal volume of PBS (pH 7.4) and analyzed for FITC-dextran concentration with a fluorescence spectrophotometer at the excitation wavelength of $485 \mathrm{~nm}$ and the emission wavelength of $535 \mathrm{~nm}$. Standard curve for calculating the FITC-dextran concentration in the samples were obtained by diluting FITC-dextran in non-treated plasma diluted with PBS (1:2).

\section{Western blot analysis}

For the immunoblotting analysis of zonula occludens-1 (ZO-1), caludin-1, and occludin, sample proteins (30 $\mu \mathrm{g}$ ) were electrophoresed through 7.5\% SDS-PAGE and transferred to polyvinylidene difluoride membranes. The transferred membrane was blocked using $2 \%$ skim milk to inhibit non-specific proteins, and treated with primary antibodies against ZO-1 (Invitrogen, Carlsbad, CA, USA), claudin-1 (Invitrogen), occludin (Invitrogen), and $\beta$-actin (Sigma-Aldrich). Anti-mouse immunoglobulin G conjugated with alkaline phosphatase was used as the secondary antibody. Each protein band was then confirmed and quantified using an enhanced chemiluminescence system (Amersham, Arlington Heights, IL, USA). The integrity of band was quantified by Versa Doc Quantity one program (BioRad, Mississauga, ON, Canada).

\section{Determination of colonic toll-like receptor 4 mRNA expression}

Total RNA was isolated from the mouse colon using Trizol reagent (Invitrogen) following the manufacture's recommendation. Reverse transcription of total RNA samples and PCR were accomplished using the premix RNA PCR kit (Invitrogen) according to the manufacturer's instruction. PCR primers were designed using nucleotide sequence for mouse toll-like receptor 4 (TLR4; Bioneer, Daejeon, Korea). The following primers were used: TLR4, forward 5'-AAT TCC TGC AGT GGG TCA AG-3' and reverse 5'-AGG CGA TAC AAT TCC ACC TG-3'; GAPDH, forward 5'-ACC TCT ATG CCA ACA CAG TGC-3' and reverse 5'-CTC ATC GTA CTC CTG CTT GC-3'. The cycling conditions were 3 minutes at $94^{\circ} \mathrm{C}, 30$ cycles of 30 seconds at $94^{\circ} \mathrm{C}, 30$ seconds at $56^{\circ} \mathrm{C}, 1$ minute at $72^{\circ} \mathrm{C}$, and 10 minutes at $72^{\circ} \mathrm{C}$. PCR products were confirmed by $2 \%$ agarose gel electrophoresis and visualized by UV transillumination (Bio-Rad Laboratories Inc., Hercules, CA, USA). For quantitative analysis, Versa Doc Image analyzer (Amersham Biosciences, Piscataway, NJ, USA) was used. All signals were normalized to the mRNA levels of the housekeeping gene $\beta$-actin and expressed as ratio.

\section{Biochemical measurement}

Serum CD14 level was determined using commercially available ELISA kit (Cell Sciences Inc., Canton, MA, USA) according to the manufacturer's instruction. Interleukin (IL)-6 level was evaluated in plasma using commercially available ELISA kit (BD Bioscience Co., Franklin Lakes, NJ, USA) according to the manufacturer's instruction. Plasma myeloperoxidase (MPO) activity was determined using commercially available ELISA kit (EIAab Science Co., Wuhan, China) according to the manufacturer's instruction.

\section{Statistical analysis}

Statistical analysis was performed by using the SAS package (release 8.01; SAS Institute, Cary, NC, USA). All data from the experiment were expressed as mean $\pm \mathrm{SD}$. Data were analyzed by 
Student's $t$-test. Differences were considered statistically significant at $P<0.05$.

\section{RESULTS}

1. Effect of high-fat diet on body weight, epididymal fat weight, liver weight, and tumor number

The body weight of HFD group was significantly higher than that of ND group between experimental week 2 and 5; however, the difference disappeared thereafter possibly due to faster tumor development in HFD group (Fig. 1A). No difference was found in liver weight and epididymal fat weight (Fig. 1B and 1C). However, the average tumor number was significantly higher in HFD mice compared to that of the ND mice ( $P<0.05$; Fig. 1D).

2. Effect of high-fat diet on blood total antioxidant capacity and colonic 8-hydroxy-2'-deoxyguanosine level

The total antioxidant capacity (TAC) is an indicator of oxidative stress. The level of 8-OHdG is one of the predominant forms of
A

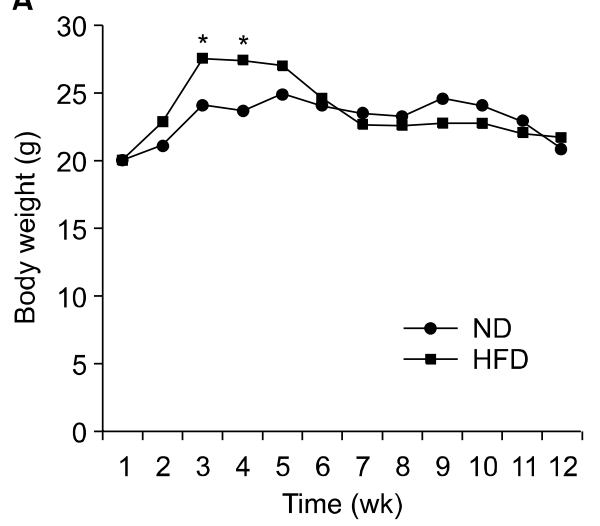

C
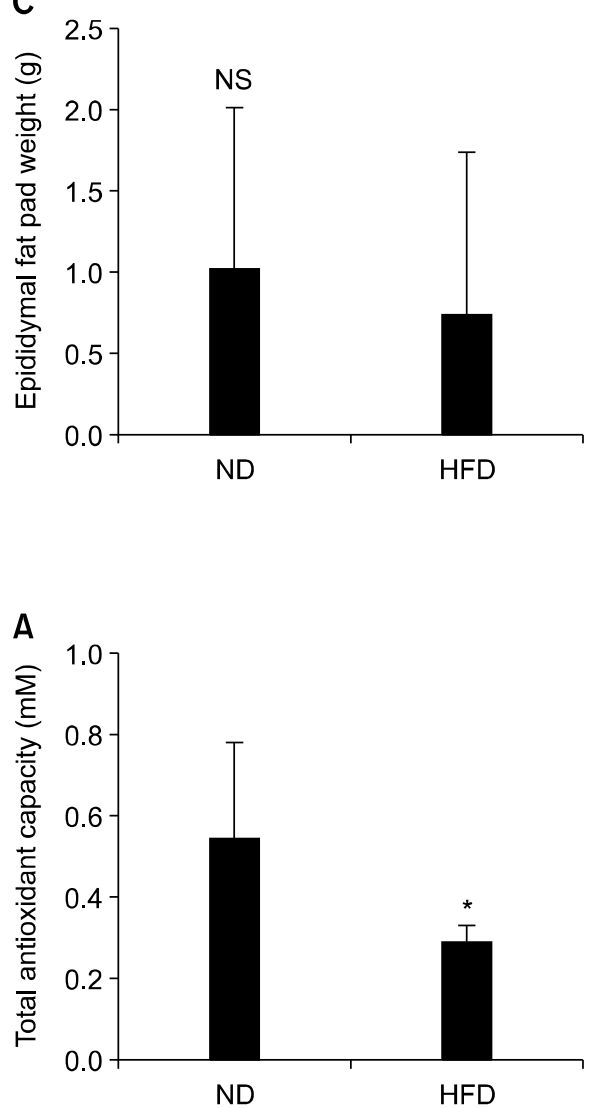

B

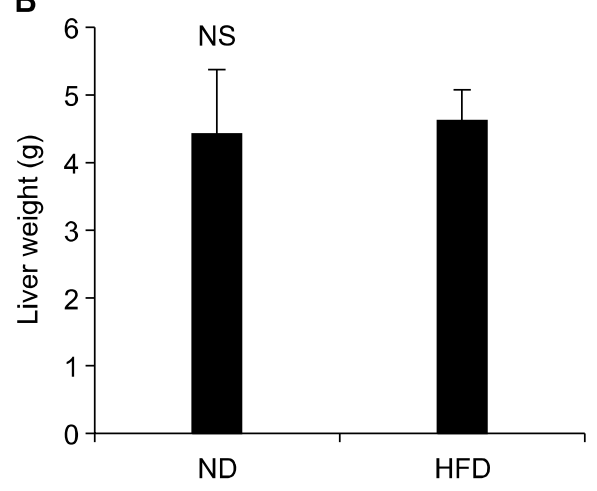

D

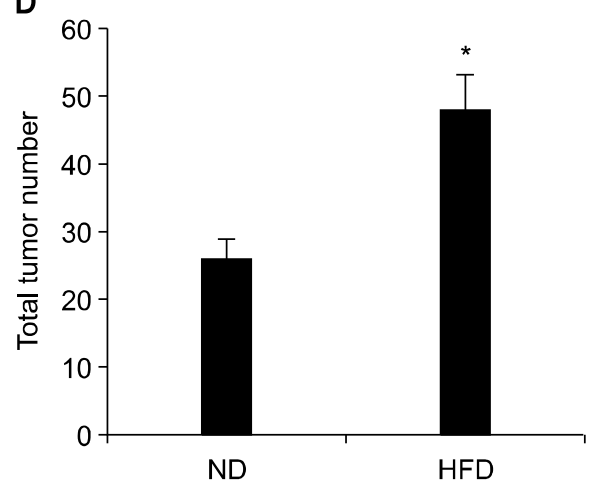

Figure 1. Effects of normal diet (ND) and high-fat diet (HFD) on body weight, tissue weight, epididymal fat pad weight, and total tumor number in $A P C^{\text {min } /+}$ mice. $A P C^{\text {min/t+ }}$ mice were fed either ND or HFD for 12 weeks. (A) Body weight, (B) liver weight, (C) epididymal fat pad weight, and (D) total tumor number was measured. Values are expressed as mean $\pm \mathrm{SD}$ (n $=10)$. *Significant $(P<0.05)$.

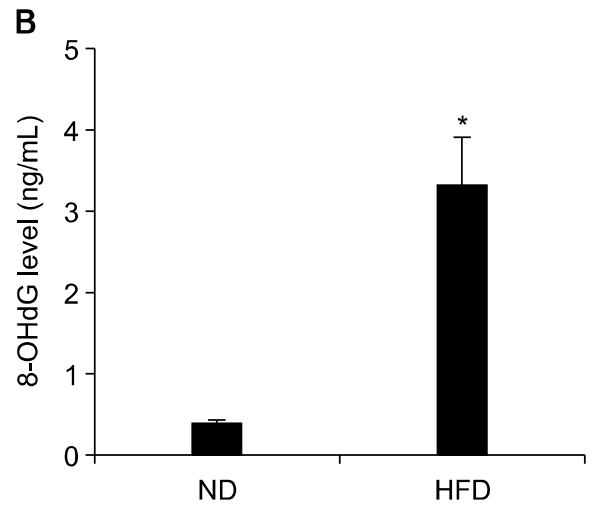

Figure 2. Effects of normal diet (ND) and high-fat diet (HFD) on blood total antioxidant capacity and colonic 8-hydroxy-2'-deoxyguanosine (8-OHdG) level in $A P C^{\min /+}$ mice. $A P C^{\min /+}$ mice were fed either ND or HFD for 12 weeks. (A) Blood total antioxidant capacity and (B) colonic 8-OHdG level were evaluated. Values are expressed as mean $\pm \mathrm{SD}(\mathrm{n}=10) . *$ Significant $(P<0.05)$. 
free radical-induced oxidative lesions in DNA which has been widely used as a biomarker for systemic oxidative stress possibly associated with carcinogenesis. Study results indicated that $A P C^{\mathrm{Min} /+}$ HFD group possessed a half TAC compared with $A P C^{\mathrm{Min} /+} \mathrm{ND}$ group, indicating that oxidative stress is significantly increased in HFD group ( $P<0.05$, Fig. 2A). In addition, 8-OHdG level in colon tissue was dramatically increased in HFD fed $A P C^{\mathrm{Min} /+}$ mice compared with tissue 8-OHdG level in ND fed $A P C^{\mathrm{Min} /+}$ mice $(P<0.05$; Fig. $2 \mathrm{~B})$. These data indicated that a substantial colonic DNA damage is induced by HFD-associated oxidative stress.

\section{Effect of high-fat diet on intestinal integrity}

Study results showed approximately a four-fold increase in serum FITC-dextran in HFD fed group, demonstrating accelerated transepithelial passage of FITC-dextran in this group $(P<0.05$; Fig. 3A). The TJ complex is a cluster of proteins that forms a physiologically active barrier at the level of the intestinal epithelial cell, changing its permeability based on the cellular environment. Key TJ proteins include ZO-1, occludin, and claudins. Since the altered paracellular permeability involves a disrupted intestinal barrier, TJ protein expressions were measured by Western blot analysis. Results showed that protein expressions of ZO-1, occludin, and claudin-1 were significantly decreased in HFD fed $A P C^{M i n /+}$ mice compared with those of ND $A P C^{\mathrm{Min} /+}$ mice $(P<0.05$; Fig. 3B 3D). These data indicate that increased intestinal permeability in HFD $A P C^{M i n /+}$ mice group might be due to the HFD-induced disruption of TJ proteins.

\section{Effect of high-fat diet on blood CD14 and colonic toll-like receptor $4 \mathrm{mRNA}$ level}

LPS is a component of the cell wall of gram-negative bacteria and is known as endotoxin. In an effort to indirectly determine endotoxemia, CD14, the LPS receptor, was measured in serum. As
A

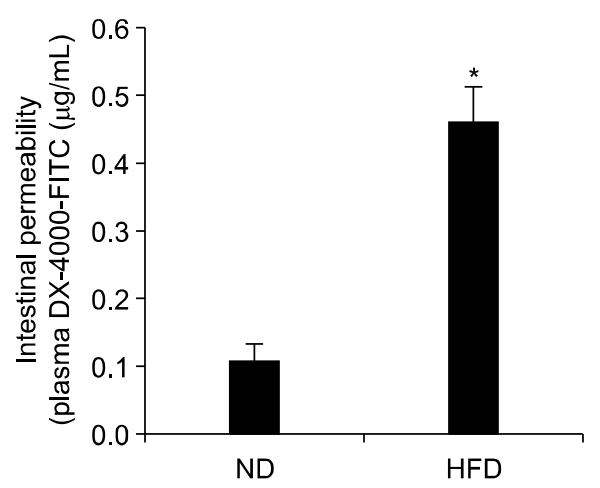

C

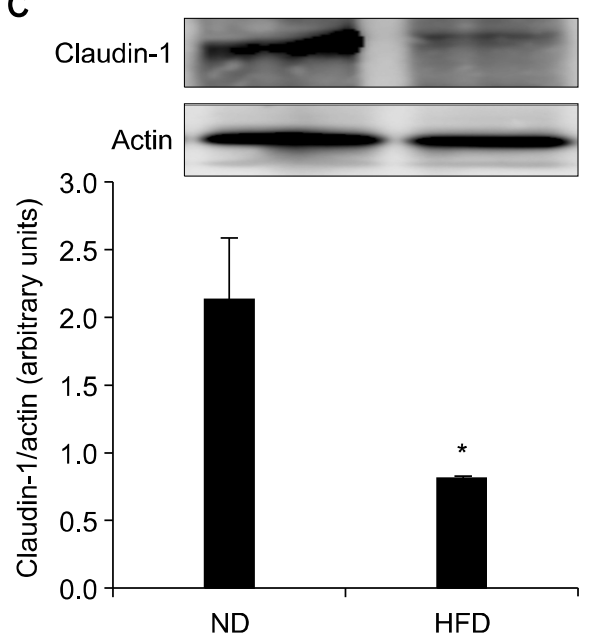

B

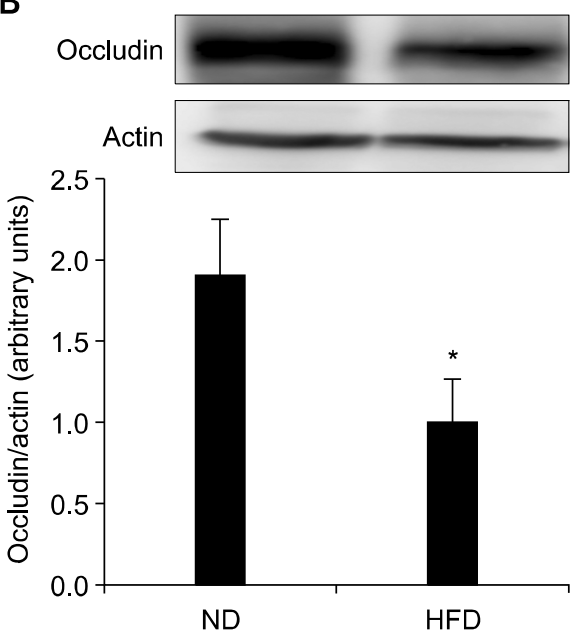

D

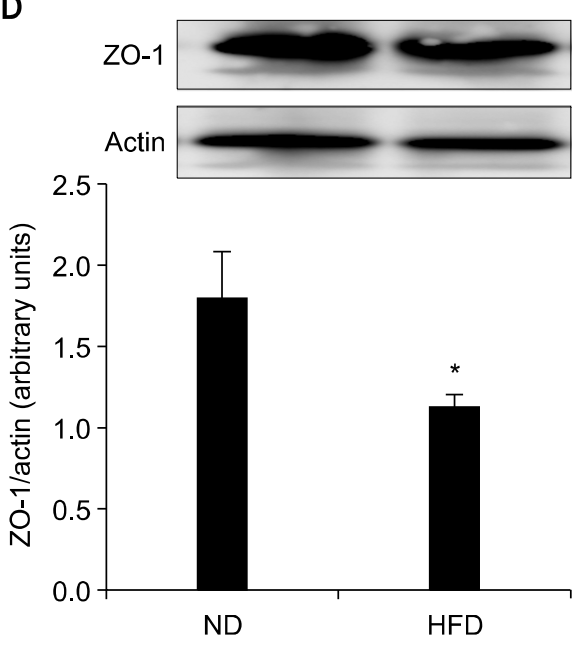

Figure 3. Effects of normal diet (ND) and high-fat diet (HFD) on intestinal permeability and colonic tight junction (TJ) protein levels in $A P C^{\min /+}$ mice. $A P C^{\min /+}$ mice were fed either ND or HFD for 12 weeks. (A) Intestinal permeability was assessed by measuring blood fluorescein isothiocyanate (FITC)-dextran level after gavage FITC-dextran $(600 \mathrm{mg} / \mathrm{kg}$ body weight). Colonic expressions of tight junction proteins, (B) occludin, (C) claudin-1, and (D) zonula occludens-1 (ZO-1), were evaluated by Western blotting. Graph shows ratios of the intensities of TJ protein bands normalized with that of $\beta$-actin band. Values are expressed as mean $\pm \mathrm{SD}(\mathrm{n}=$ 10). *Significant $(P<0.05)$. 
LPS is a ligand of TLR4 in the epithelium, the mRNA expression of TLR4 was determined in colon tissue. Figure 4 showed that $A P C^{\mathrm{Min} /+}$ mice fed HFD had significantly higher concentration of serum CD14 than mice fed ND ( $P<0.05$; Fig. 4A). A two-fold increase mRNA expression of TLR4 in colon tissue was also observed in $A P C^{M i n /+}$ mice fed HFD ( $P<0.05$; Fig. 4B).

\section{Effect of high-fat diet on blood interleukin-6 level and myeloperoxidase activity}

Plasma IL-6 level and MPO level are shown in Figure 5. Plasma IL-6 concentration was significantly increased in $A P C^{M i n /+}$ mice fed HFD ( $P<0.05$; Fig. 5A). MPO level, an index of granulocytic infiltration, was also significantly increased in the HFD fed $A P C^{\mathrm{Min} /+}$ mice $(P<0.05$; Fig. $5 \mathrm{~B})$. These results indicate that HFD significantly accelerates inflammatory response in $A P C^{\mathrm{Min} /+}$ mice which is possibly linked to tumorigenesis.

\section{DISCUSSION}

The aim of this study was to provide mechanistic insights for HFD mediated intestinal tumorigenesis. Three weeks after the experimental diet began, animals on HFD started to gain significantly more weight. However, differences in body weight between groups became insignificant starting at week 6. Mice in both experimental groups may have developed polyps as we observed bloody stools. Tumor-induced weight loss is a common feature of cancer and is caused by wasting of muscle and adipose tissue. Depletion of body fat is caused by either the inhibition of the lipoprotein lipase or the stimulation of triglyceride hydrolysis. ${ }^{22}$ A decreased rate of protein synthesis and enhanced protein degradation also contributed to protein depletion. These may result in the loss of body weight as they start to bear polyps. Concurrently, HFD itself can remain to directly affect the integrity of intestinal epithelium by generating ROS followed by creating inflammatory environment. Previous studies have also indicated that the increased production of secondary bile acids
A

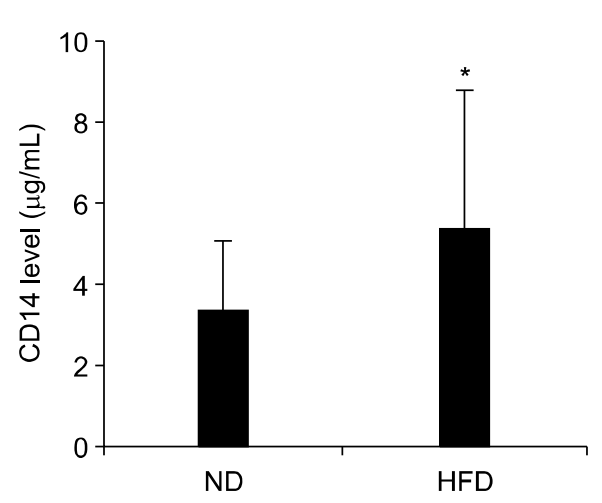

A

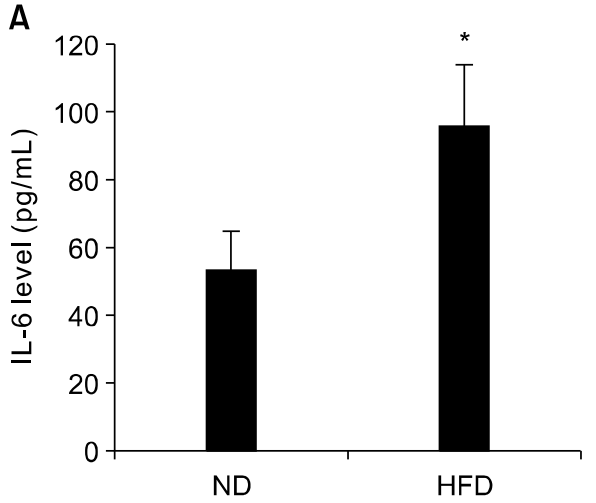

B

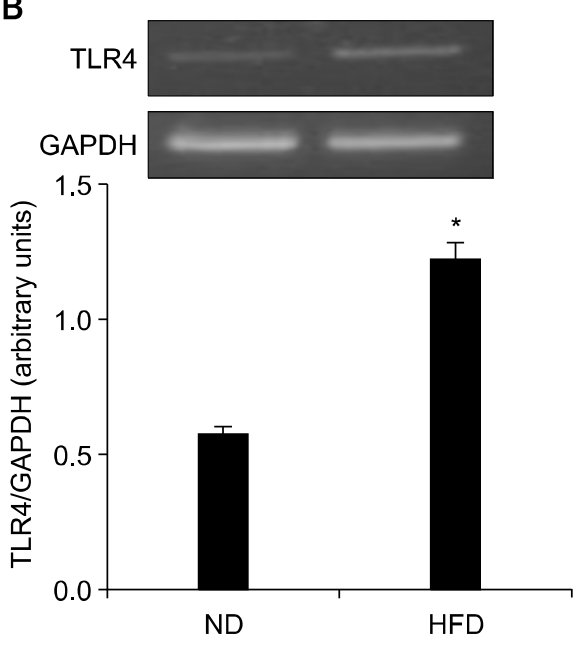

Figure 4. Effects of normal diet (ND) and high-fat diet (HFD) on blood CD14 level and colonic toll-like receptor 4 (TLR4) mRNA expression levels in $A P C^{\min / t}$ mice. $A P C^{\text {min/t }}$ mice were fed either ND or HFD for 12 weeks. (A) CD14 level in blood was measured by commercially available ELISA kit. (B) Colonic TLR4 gene expression level was evaluated by RT-PCR. Graph shows ratios of TLR4 band intensity normalized with $\beta$-actin band. Values are expressed as mean $\pm \mathrm{SD}(\mathrm{n}=10)$. GAPDH, glyceraldehyde-3-phosphate dehydrogenase. *Significant $(P<0.05)$.

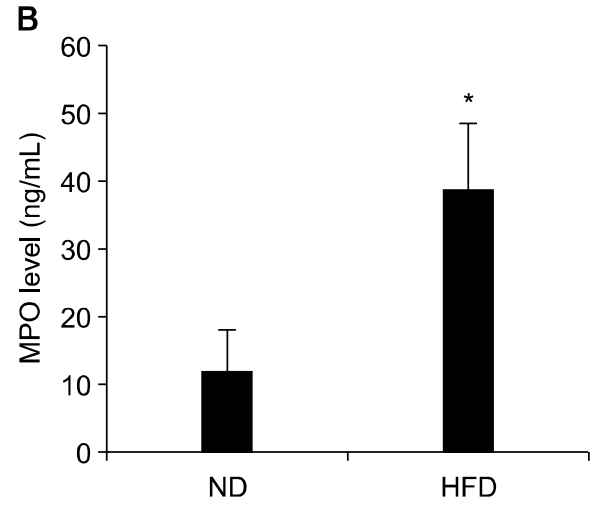

Figure 5. Effects of normal diet (ND) and high-fat diet (HFD) on blood interleukin (IL)-6 level and myeloperoxidase (MPO) level in $A P C^{\text {min } /+}$ mice. $A P C^{\mathrm{min} /+}$ mice were fed either ND or HFD for 12 weeks. Blood (A) IL-6 and (B) MPO levels were measured by commercially available ELISA kit. Values are expressed as mean $\pm \mathrm{SD}$ $(\mathrm{n}=10) . *$ Significant $(P<0.05)$. 
accelerates the colon tumor formation. ${ }^{23}$

It is reported that increased fat storage is linked with increased generation of ROS. ${ }^{24}$ ROS can interact with DNA to produce damage including single and double-stranded DNA breaks and nucleotide modifications. The level of 8-OHdG, the oxidized form of the nucleoside 2'-deoxyguanosine present in DNA, is one of the most reliable and abundant markers for free radical-induced oxidative lesions. ${ }^{25}$ Study results indicated that total antioxidant capacity was lower in the HFD group than those of the ND group, while 8-OHdG level was higher in the HFD group. These results show that mice fed HFD were under a heavier oxidative stress and antioxidant defenses might be weakened than mice fed ND.

ROS may also be one of the contributor in gut dysfunction. ${ }^{26}$ Increased ROS can rapidly stimulate compartmental redistribution of TJs such as occludin and ZO-1 in Caco-2 cells. ${ }^{27}$ The disruption of TJs plays an important role in the pathogenesis of a number of gastrointestinal diseases including inflammatory bowel disease, celiac disease, allergy, and cancer. ${ }^{27,28}$ In this study, HFD remarkably increased passage of FITC-dextran and significantly decreased expression levels of $\mathrm{TJ}$ proteins including occludin, ZO-1, and claudin-1. A previous study has indicated that HFD causes intestinal eosinophil depletion which could result in defective barrier function. ${ }^{29}$ Not only to promote intestinal tumorigenesis, but severe gut barrier dysfunction enhances progression of colon cancer cachexia in the $\mathrm{ApC}^{\mathrm{Min} /+}$ mice model through increased endotoxemia and higher circulating concentration of IL- 6 . $^{30}$ Increased intestinal permeability permits diffusion of bacterial endotoxins such as LPS into the blood stream which is associated with systemic inflammation and development of several diseases. ${ }^{20,31}$ Previous reports showed that inflammation markers were decreased in ob/ob mice lacking LPS receptor CD14 and identified HFD-induced LPS is a triggering factor in metabolic endotoxemia. ${ }^{20,32}$ In a case-control study, blood endotoxin levels were shown to be correlated with colon polyps. ${ }^{33}$ We clearly found that HFD significantly increased blood CD14 level and colonic TLR4 mRNA expression level. Among several TLRs, TLR4 is stimulated by binding bacterial LPS. The TLR4/NF-KB signaling pathway is known to stimulate the secretion of inflammatory cytokines including IL-6 and IL-8, and TLR4-dependent inflammatory response contributed to the colorectal cancer (CRC) development. ${ }^{34}$ A previous study also showed that the up-regulated TLR4 and its polymorphism are associated with progression of colon cancer. ${ }^{35}$ TLR4 was also suggested as an important CRC specific cell-surface marker in colorectal cancer patients. $^{36}$ As LPS treatment increases paracellular permeability in intestinal epithelial cells, ${ }^{37}$ it can be postulated that HFD facilitates LPS production and LPS-induced signaling causes endotoxemia, resulting in increased intestinal permeability possibly accelerating CRC progression.

In this study, serum IL-6 concentration and MPO level were used as inflammatory markers. Among several inflammatory cytokines, IL-6 is known as a predictive marker for CRC progression. ${ }^{38-40} \mathrm{MPO}$ is a specific marker of neutrophils infiltration, which can be considered as an inflammatory damage index. ${ }^{41,42}$ As expected, both serum IL-6 and MPO level were elevated in the HFD group compared to the ND group in accordance with increased permeability markers.

In summary, HFD induced oxidative stress and endotoxemia, leading to disruption of intestinal barrier in $A P C^{\mathrm{Min} /+}$ mice. In addition, HFD increased both oxidative stress and LPS-related markers. These results indicate that increased intestinal permeability allows entrance of bacterial pathogens and may cause chronic inflammation which accelerates the formation of intestinal polyps in $A P C^{M i n /+}$ mice. This study is one of a few studies suggesting that HFD influences intestinal tumorigenesis by increasing intestinal permeability via oxidative stress and endotoxemia.

\section{ACKNOWLEDGMENTS}

This work was supported by the High Value-added Food Technology Program (grant number 312006-3) funded by the Ministry of Agriculture, Food and Rural Affairs and by the Mid-Career Research Program (2015R1A2A2A01004607) of the National Research Foundation of Korea funded by the Ministry of Science, ICT and Future Planning.

\section{CONFLICTS OF INTEREST}

No potential conflicts of interest were disclosed.

\section{REFERENCES}

1. World Health Organization (WHO), 2013. Cancer Incidence and Mortality Worldwide: IARC. http://www.who.int/mediacentre/ factsheets/fs297/en/. Accessed June 13, 2015.

2. Tárraga López PJ, Albero JS, Rodríguez-Montes JA. Primary and secondary prevention of colorectal cancer. Clin Med Insights Gastroenterol 2014:7:33-46.

3. Ames BN. DNA damage from micronutrient deficiencies is likely to be a major cause of cancer. Mutat Res 2001;475:7-20.

4. Ju J, Liu Y, Hong J, Huang MT, Conney AH, Yang CS. Effects of green tea and high-fat diet on arachidonic acid metabolism and aberrant crypt foci formation in an azoxymethane-induced colon 
carcinogenesis mouse model. Nutr Cancer 2003:46:172-8.

5. Laugerette F, Vors C, Peretti N, Michalski MC. Complex links between dietary lipids, endogenous endotoxins and metabolic inflammation. Biochimie 2011;93:39-45.

6. Dandona P, Ghanim H, Chaudhuri A, Dhindsa S, Kim SS. Macronutrient intake induces oxidative and inflammatory stress: potential relevance to atherosclerosis and insulin resistance. Exp Mol Med 2010;42:245-53.

7. Nikolaidis MG, Kerksick CM, Lamprecht M, McAnulty SR. Redox biology of exercise. Oxid Med Cell Longev 2012;2012:407978.

8. Gutierrez-Lopez L, Garcia-Sanchez JR, Rincon-Viquez Mde J, Lara-Padilla E, Sierra-Vargas MP, Olivares-Corichi IM. Hypocaloric diet and regular moderate aerobic exercise is an effective strategy to reduce anthropometric parameters and oxidative stress in obese patients. Obes Facts 2012;5:12-22.

9. Al-Aubaidy HA, Jelinek HF. Oxidative DNA damage and obesity in type 2 diabetes mellitus. Eur J Endocrinol 2011;164:899-904.

10. Mena S, Ortega A, Estrela JM. Oxidative stress in environmental-induced carcinogenesis. Mutat Res 2009;674:36-44.

11. Valko M, Rhodes CJ, Moncol J, Izakovic M, Mazur M. Free radicals, metals and antioxidants in oxidative stress-induced cancer. Chem Biol Interact 2006; 160:1-40.

12. Perše M. Oxidative stress in the pathogenesis of colorectal cancer: cause or consequence? Biomed Res Int 2013;2013:725710.

13. Foksinski M, Rozalski R, Guz J, Ruszkowska B, Sztukowska P, Piwowarski $\mathrm{M}$, et al. Urinary excretion of DNA repair products correlates with metabolic rates as well as with maximum life spans of different mammalian species. Free Radic Biol Med 2004:37:1449-54.

14. Lemanowicz A, Serafin Z. Imaging of patients treated with bariatric surgery. Pol J Radiol 2014;79:12-9.

15. Wang JH, Bose S, Kim GC, Hong SU, Kim JH, Kim JE, et al. Flos lonicera ameliorates obesity and associated endotoxemia in rats through modulation of gut permeability and intestinal microbiota. PLoS One 2014;9:e86117.

16. Duca FA, Sakar Y, Lepage P, Devime F, Langelier B, Doré J, et al. Replication of obesity and associated signaling pathways through transfer of microbiota from obese-prone rats. Diabetes 2014:63:1624-36.

17. Neves AL, Coelho J, Couto L, Leite-Moreira A, Roncon-Albuquerque R Jr. Metabolic endotoxemia: a molecular link between obesity and cardiovascular risk. J Mol Endocrinol 2013;51:R51-64.

18. Bruewer M, Luegering A, Kucharzik T, Parkos CA, Madara JL, Hopkins AM, et al. Proinflammatory cytokines disrupt epithelial barrier function by apoptosis-independent mechanisms. J Immunol 2003; 171:6164-72.

19. Das UN. Is obesity an inflammatory condition? Nutrition 2001:17:953-66

20. Cani PD, Bibiloni R, Knauf C, Waget A, Neyrinck AM, Delzenne $\mathrm{NM}$, et al. Changes in gut microbiota control metabolic endotoxemia-induced inflammation in high-fat diet-induced obesity and diabetes in mice. Diabetes 2008:57:1470-81.

21. Su LK, Kinzler KW, Vogelstein B, Preisinger AC, Moser AR, Luongo C, et al. Multiple intestinal neoplasia caused by a mutation in the murine homolog of the APC gene. Science 1992;256:668-70.

22. Tisdale MJ. Wasting in cancer. J Nutr 1999;129:243S-6S.

23. Cao H, Luo S, Xu M, Zhang Y, Song S, Wang S, et al. The secondary bile acid, deoxycholate accelerates intestinal adenoma-ad- enocarcinoma sequence in Apc $(\mathrm{min} /+)$ mice through enhancing Wnt signaling. Fam Cancer 2014;13:563-71.

24. Wu Q, Ni X. ROS-mediated DNA methylation pattern alterations in carcinogenesis. Curr Drug Targets 2015;16:13-9.

25. Choi YJ, Seelbach MJ, Pu H, Eum SY, Chen L, Zhang B, et al. Polychlorinated biphenyls disrupt intestinal integrity via NADPH oxidase-induced alterations of tight junction protein expression. Environ Health Perspect 2010;118:976-81.

26. Catanzaro D, Rancan S, Orso G, Dall'Acqua S, Brun P, Giron MC, et al. Boswellia serrata preserves intestinal epithelial barrier from oxidative and inflammatory damage. PLoS One 2015;10:e0125375.

27. Merga Y, Campbell BJ, Rhodes JM. Mucosal barrier, bacteria and inflammatory bowel disease: possibilities for therapy. Dig Dis 2014:32:475-83.

28. Lu Z, Ding L, Lu Q, Chen YH. Claudins in intestines: distribution and functional significance in health and diseases. Tissue Barriers 2013;1:e24978.

29. Johnson AM, Costanzo A, Gareau MG, Armando AM, Quehenberger O, Jameson JM, et al. High fat diet causes depletion of intestinal eosinophils associated with intestinal permeability. PLoS One 2015;10:e0122195.

30. Puppa MJ, White JP, Sato S, Cairns M, Baynes JW, Carson JA. Gut barrier dysfunction in the $\mathrm{Apc}(\mathrm{Min} /+)$ mouse model of colon cancer cachexia. Biochim Biophys Acta 2011;1812:1601-6.

31. Swank GM, Deitch EA. Role of the gut in multiple organ failure: bacterial translocation and permeability changes. World J Surg 1996;20:411-7.

32. Cani PD, Amar J, Iglesias MA, Poggi M, Knauf C, Bastelica D, et al. Metabolic endotoxemia initiates obesity and insulin resistance. Diabetes 2007:56:1761-72.

33. Lee KK, Yum KS. Association of endotoxins and colon polyp: a case-control study. J Korean Med Sci 2012;27:1062-5.

34. Huang HY, Zhang ZJ, Cao CB, Wang N, Liu FF, Peng JQ, et al. The TLR4/NF- $\mathrm{KB}$ signaling pathway mediates the growth of colon cancer. Eur Rev Med Pharmacol Sci 2014;18:3834-43.

35. Omrane I, Baroudi O, Kourda N, Bignon YJ, Uhrhammer N, Desrichard A, et al. Positive link between variant Toll-like receptor 4 (Asp299Gly and Thr399Ile) and colorectal cancer patients with advanced stage and lymph node metastasis. Tumour Biol 2014:35:545-51.

36. Sewda K, Coppola D, Enkemann S, Yue B, Kim J, Lopez AS, et al. Cell-surface markers for colon adenoma and adenocarcinoma [published online ahead of print February 15, 2016]. Oncotarget. doi: 10.18632/oncotarget.7402.

37. Bein A, Zilbershtein A, Golosovsky M, Davidov D, Schwartz B. LPS induces hyper-permeability of intestinal epithelial cells [published online ahead of print May 18, 2016]. J Cell Physiol. doi: 10.1002/jcp. 25435 .

38. Shimazaki J, Goto Y, Nishida K, Tabuchi T, Motohashi G, Ubukata $\mathrm{H}$, et al. In patients with colorectal cancer, preoperative serum interleukin-6 level and granulocyte/lymphocyte ratio are clinically relevant biomarkers of long-term cancer progression. Oncology 2013;84:356-61.

39. Lu CC, Kuo HC, Wang FS, Jou MH, Lee KC, Chuang JH. Upregulation of TLRs and IL-6 as a marker in human colorectal cancer. Int J Mol Sci 2014;16:159-77.

40. Lee WS, Baek JH, You DH, Nam MJ. Prognostic value of circulating cytokines for stage III colon cancer. J Surg Res 2013;182:49-54. 
41. Zheng L, Gao ZQ, Wang SX. A chronic ulcerative colitis model in rats. World J Gastroenterol 2000;6:150-2.

42. Gambero A, Maróstica M, Abdalla Saad MJ, Pedrazzoli J Jr.
Mesenteric adipose tissue alterations resulting from experimental reactivated colitis. Inflamm Bowel Dis 2007;13:1357-64. 